Research Article

\title{
A Novel Threshold - Controllable Algorithm for Moving Target Coverage
}

\author{
Guozeng Zhao ${ }^{1, *}$, Hengchuan Guo ${ }^{1}$, Zeyu Sun ${ }^{1}$ and Weidong Zheng ${ }^{1,2}$ \\ ${ }^{1}$ School of Computer and Information Engineering, Luoyang Institute of Science and Technology, Luoyang 471023, China \\ ${ }^{2}$ Department of Control and Computer Engineering, Politecnico di Torino, Corso Duca degli Abruzzi 24, 10129 Torino, Italy
}

Received 1 December 2017; Accepted 22 March 2018

\begin{abstract}
The uncertainty of a moving target's speed and direction can lead to inaccurate target location and large energy consumption of network nodes when the target is covered and controlled by the sensor node in the wireless sensor network. This study proposed a method of threshold-controllable moving target coverage to accurately predict the target movement location, improve the energy consumption rate of the network, and clarify the main factors affecting the coverage of the moving target. First, a node participation threshold was introduced in the area of the moving target, and a scheduling mechanism of the node state was established. Second, the location prediction model of the moving target and the self-adjusting mechanism of the node data reporting frequency were established in accordance with the historical location information, movement direction, and speed of the moving target. Finally, the effect of node participation threshold on target location accuracy and network energy consumption during monitoring of the moving target was analyzed. Results show that when the participation threshold is set to decrease from 5 to 2 , the target location error at different speeds decreases by $56.7 \%$. When the participation threshold is equal to 3 , the average energy consumption at different speeds decreases by 55\% compared with consumption without a present threshold. Energy consumption decreases by an average of $41 \%$ in the model of moving target location prediction. Therefore, the threshold-controllable algorithm for moving target coverage shows excellent performance in terms of location accuracy and network energy utilization efficiency. The study can be utilized for sensor network environments with limited node energy to provide theoretical guidance for the dynamic coverage optimization of moving targets.
\end{abstract}

Keywords: Moving target, Coverage, Threshold-controllable, Location prediction, Dynamic coverage group

\section{Introduction}

Wireless sensor networks are self-organizing networks formed by data acquisition, storage, processing, wireless communication, and low-power sensor nodes through wireless communication protocols that are based on sensors, networks, and microelectromechanical systems. Wireless sensor networks are widely applied in military monitoring, disaster rescue, environmental monitoring, intelligent transportation, medical treatment, and health. As a basic research aspect on wireless sensor networks, coverage technology fundamentally reflects a sensor network's perception of the physical world with the help of sensor perception, node configuration, and target monitoring to completely and effectively acquire the needed information [1].

At present, the coverage algorithm for static wireless sensor networks is relatively mature, whereas that for moving targets is less developed [2, 3]. In practice, wireless sensor networks are mainly used in the dynamic monitoring of sensor nodes about moving targets within a coverage area; examples of such applications include vehicle tracking in military monitoring and wildlife tracking in habitat monitoring [4]. The coverage of a moving target must be capable of effectively aiming at the target perception,

\footnotetext{
*E-mail address: ly_zgz@163.com

ISSN: 1791-2377 @ 2018 Eastern Macedonia and Thrace Institute of Technology. All rights reserved. doi:10.25103/iestr.112.01
}

location, and tracking, in addition to flexible scheduling of nodes for the moving target at different speeds and different locations. The power of sensor nodes is restricted by the limited battery power of sensor nodes, and tracking quality and network life are contradictory. Therefore, network life extension and tracking quality guarantee are important issues in moving target monitoring research, particularly in the coverage of moving targets $[5,6]$.

Network nodes must be reasonably scheduled to allow continuous monitoring of moving targets by the wireless sensor network while reducing the network energy consumption. The wireless sensor network predicts the possible location of a moving target in a future time period. The network also schedules the nodes near the location to participate in monitoring the moving target on the basis of the moving target's movement locus. Thus, the energy consumption of unnecessary nodes can be effectively diminished to improve the energy utilization efficiency of nodes in the entire network.

Given the above discussion, this study established the moving target coverage model by dynamically adjusting the node participation threshold of a moving target's speed to improve the location accuracy of sensor nodes and the utilization of network energy.

\section{State of the art}

In the past several years, moving target tracking in wireless sensor networks has been closely investigated. The earliest 
research on the coverage of moving targets started with a study on barrier coverage [7, 8]. With the in-depth application of sensor networks, users' coverage monitoring of moving targets proposed new requirements for target location accuracy, node energy consumption efficiency, and real-time monitoring capability. However, numerous studies only focused on the location accuracy of desired targets without focusing on network energy consumption [9-11].

To date, a large number of foreign and domestic scholars have investigated these problems and proposed solutions. Zebbane et al. [12] reduced energy consumption by controlling the size of ethnic groups but failed to predict the moving target. Simultaneously, multiple nodes were in an internal state of arousal to prevent target loss; as a result, substantial energy consumption was wasted. By optimizing the traditional distributed fusion structure through the use of an averaging filter, Li et al. [13] effectively completed the prediction of movement trend but failed to optimize the node state, thereby causing energy consumption waste. Liu et al. [14] proposed the strategy of self-adaptive dynamic cluster target tracking with relatively high location accuracy and lowered communication overhead. However, a satisfactory level was not reached. Ren et al. [15] divided the total dynamic coverage process into two stages. The first stage involves data acquisition and perceived findings, whereas the second stage includes target location and tracking. Different sleep scheduling mechanisms were adopted on the basis of different requirements of node density at different stages. Monitoring node selection was transformed into the problem of network coverage with an approximate given solution. Concurrently, the Markov chain model was used, and the matrix of transition probability was established to predict the node in charge of monitoring at the next moment and trigger the node in advance. The monitoring quality of a moving target is effectively improved, but the parameter selection of the moving target dynamic coverage model in this method is based on the empirical value; therefore, with inappropriate parameter selection, the moving target location incurs a large error. Jeong et al. [15] proposed a moving target dynamic coverage algorithm based on vehicle movement locus. In this method, the most likely and unlikely areas that the vehicle may reach were extracted within a finite time range; thus, the tracking range of the sensor node and the number of nodes involved in tracking and communication traffic in the network were reduced to extend the life cycle of the wireless sensor network [16]. This strategy exhibits an effective energy-saving property for vehicle monitoring in a steady motion state but easily causes target "loss" for target nodes with unstable motion states [17]. Sun et al. [18] proposed the target location and dynamic coverage algorithm based on distance weighting; this method acquires the distance between the monitoring node and the target node through the received signal strength and introduces weight depending on distance. This technique improves the location speed of the target node, but does not consider the optimization problem of selecting the node participating in the tracking. Zhang et al. [19] adopted a node cooperation framework based on tree structure and proposed a dynamic coverage method for moving targets. Under this strategy, effective dynamic coverage of target nodes is achieved by dynamically adding or deleting nodes in the tree to constantly reconstruct the tree structure [20,21]. However, during project implementation, the large scale of dynamic tree nodes produces low-quality and redundant target data, which increases the communication and system overhead of the network [22].
Existing domestic and foreign studies have explored the coverage of moving targets. However, the following disadvantages persist. First, coverage of a moving target mainly focuses on improving the location accuracy of a moving target or effectively reducing the network energy consumption; however, this approach disregards the two factors simultaneously. Second, the monitoring node cannot flexibly adjust its monitoring depending on the change of the target's moving speed with regard to the coverage strategy.

Therefore, given such drawbacks, this study adjusted different node participation thresholds on the basis of target moving speed to construct a dynamic coverage group in real time, predict location, and thus improve the location accuracy and energy utilization rate of target node monitoring. The present work also established the selfadjusting mechanism of node data reporting frequency on the basis of the moving target speed to improve the utilization rate of the network energy consumption.

The remainder of this study is organized as follows. Section 3 establishes the moving target coverage model. Section 4 sets up the experimental evaluation indicators and analyzes the experimental results. Section 5 summarizes this study and provides the relevant conclusion.

\section{Methodology}

\subsection{Model hypothesis}

To thoroughly study the dynamic coverage of a moving target, we introduce four hypotheses on the wireless sensor network characteristics [23, 24]

Hypothesis 1 Sensor nodes are distributed randomly in the target area.

Hypothesis 2 The perceived radius and communication radius of sensor nodes are equal to those of the homogeneous sensor network.

Hypothesis 3 Nodes adopt a circular perception and a communication model with location as the circle center. Hypothesis 4 Sensor nodes can automatically acquire their own location information.

\subsection{Basic concept}

For improving the fault tolerance of the network, sensor nodes are usually distributed densely in the target area after the initial deployment of network nodes. The total target area is divided into several of grids, and the sensor nodes are added to the corresponding grids in accordance with their locations. This step was applied to decrease the unnecessary energy consumption of nodes. In each grid, a node is elected as a cluster head, and the remaining nodes are member nodes. The cluster head node takes charge of the management of nodes in the entire area (Figure 1). After monitoring the moving target, the cluster head node in active state broadcasts the message to member nodes, and each node calculates the weight of the participating target node with the help of message passing by the neighboring nodes. When the weight exceeds the set threshold $w_{\text {ths }}$, the node is transferred to active state to participate in the monitoring of the target node. In this manner, a dynamic coverage group led by cluster head nodes is formed near the moving target. As the target node moves in the monitoring area, the possible area that the target node may reach is projected using the prediction model of the target node. The cluster head node is triggered in the next possible area by the current cluster head node, and the node is scheduled in the next area in the 
waiting state to improve the real-time capacity of moving target node monitoring and diminish moving target loss. Given the size of the target moving speed, the node may fail to monitor the target in time or the node may frequently monitor the target excessively if the current cluster head node reports data at a fixed frequency. Therefore, cluster head nodes utilize the self-adjusting mechanism of data reporting frequency to send the target information data after fusion to the base station.

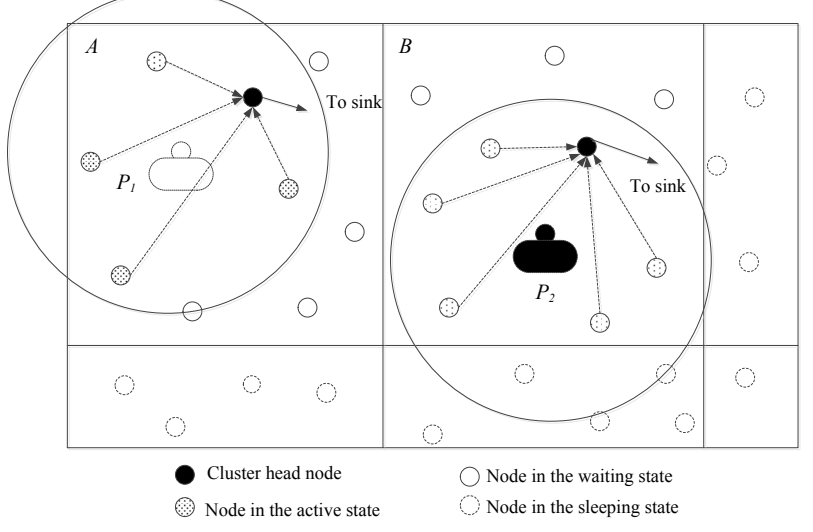

Fig. 1. Moving target from $\mathrm{P} 1$ to $\mathrm{P} 2$

\subsection{Calculation of node weight involved in dynamic coverage}

When the moving target shifts in the monitoring area, monitoring of the participation of the moving target node should be minimized, and the internet life cycle should be improved. Initially, the node away from the moving target is interfered by magnetic field and noise, and signal attenuation increases during broadcast. Consequently, the data quality of the monitoring target acquired by the node is relatively low. Therefore, the dynamic monitoring of the node away from the target should be reduced to the maximum extent.

Definition 1 (distance weight): Suppose that the coordinate value of the target node $t_{i}$ is $\left(x_{t}, y_{t}\right)$; the coordinate value of any sensor node $s_{i}$ is $\left(x_{t}, y_{t}\right)$, and the distance weight $w_{\text {dis }}$ of the sensor node $s_{i}$ against the target node $t_{i}$ is:

$w_{d i s}=\left\{\begin{array}{cc}1, & d\left(s_{i}, t_{i}\right)>r_{s} \\ 2, & \frac{2}{3} r_{s}<d\left(s_{i}, t_{i}\right) \leq r_{s} \\ 3, & \frac{1}{3} r_{s}<d\left(s_{i}, t_{i}\right) \leq \frac{2}{3} r_{s} \\ 4, & d\left(s_{i}, t_{i}\right) \leq \frac{1}{3} r_{s}\end{array}\right.$

Where $r_{s}$ is the sensory distance of the sensor node.

Second, when the target moves away from the node, the quality of data acquired by the node gradually decreases, and the node achieves a relatively low coverage participation right. By contrast, when the target moves to the node, the quality of data acquired by the node gradually improves. In this case, the coverage participation at the right of the node is enhanced.

Definition 2 (distance weight): Suppose that the $d\left(s_{i}, t_{i}\right)$ distance is reduced and the weight is increased when the target node $t_{i}$ moves to the direction of participation node $s_{i}$; the $d\left(s_{i}, t_{i}\right)$ value increases, and the weight is reduced when the target node $t_{i}$ moves away from the direction of participation node $s_{i}$. The distance weight $w_{d i r}$ is defined as follows:

$w_{\text {dir }}= \begin{cases}1, & d\left(s_{i}, t_{i}\right) \uparrow \\ 0, & d\left(s_{i}, t_{i}\right) \text { unchanged } \\ -1, & d\left(s_{i}, t_{i}\right) \downarrow\end{cases}$

Definition 3 (node weight): Suppose that the distance weight of the participation node is $w_{d i s}$ and the direction weight is $w_{d i r}$; then the weight of the participation dynamic coverage node $w$ generally considers the two factors:

$w=w_{d i s}+w_{d i r}$

By setting different weights, the node obtains the maximum participation weight when the distance between the sensor node and the target is less than $\frac{1}{3} R_{s}$ and the target moves toward the node. This result indicates that the node achieves the optimal data monitoring capability under the circumstances. On the contrary, when the distance between the sensor node and the target exceeds the sensory distance $R_{s}$ and the target moves toward the direction away from the node, then the participation weight of the node is 0 . This result indicates that the node involved in the monitoring is illogical. To quantify whether the nodes with different weights participated in the target monitoring, we set a threshold $w_{\text {ths }}$ of node participation monitoring and stipulated that only the node with a value exceeding or equal to the node threshold is allowed to participate in target monitoring. A large set threshold means that a low number of nodes can participate in target monitoring. Conversely, a large number of nodes can participate in the target monitoring when a low threshold is set. Therefore, the size of the set threshold is related to the target location accuracy of a node and the network energy consumption.

\subsection{Node state scheduling mechanism}

To improve the node energy utilization efficiency, the node is divided into three working states, namely, sleeping, waiting, and active. The node state scheduling mechanism is as follows:

(1) Under the initial network condition, the cluster head nodes are in the active state, and the member nodes are in the sleeping state.

(2) Nodes in the sleeping state are periodically awakened to check whether an on-duty message is sent by the cluster head nodes. If the on-duty message is received, then the nodes in the sleeping state shift to the waiting state. Otherwise, the nodes remain in the sleeping state.

(3) The weight size of the nodes that participate in the target monitoring in the waiting state is periodically calculated. If the participation monitoring weight exceeds or is equal to the threshold, then the node shifts to the active state to participate in the monitoring of the moving target. If an off-duty message sent by the cluster head node is received, 
then the node shifts to the sleeping state.

(4) The weight size of the nodes that participate in the target monitoring in the active state is periodically calculated. If the calculated weight is less than the threshold, then the node shifts to the waiting state; otherwise, the node continues to monitor the moving target in the active state.

\subsection{Location prediction model of moving target}

Given the moving speed change of the moving target, delay in data communication between nodes, and the moving target location time overhead of the node, the location error of the node for the moving target rapidly increases, and even the "loss" of moving target occurs in extreme cases. To enable the node to monitor the moving target in time and avoid the "loss" of target monitoring, the system must trigger the node in the area in which the moving target is expected to move.

To monitor the target in the perception area in time, this study proposed the target location prediction model (TLPM). The main function of TLPM is to estimate the next possible moving monitoring area by interpolation on the basis of the node's historical location information and the current moving direction and speed of the moving target. The interpolation formula of $n^{\text {th }}$-order equal distance is as follows:

$$
\begin{aligned}
\hat{x}_{t}= & x_{t-n-1}+(n+1) \mathrm{V} x_{t-n-1}+\frac{(n+1) n}{2 !} \mathrm{V}^{2} x_{t-n-1} \\
& +\cdots+\frac{(n+1) n \cdots 2}{n !} \mathrm{V}^{n} x_{t-n-1}
\end{aligned}
$$

Where $(n+1) \mathrm{V} x_{t-n-1}$ is the first-order difference, $\mathrm{V}^{2} x_{t-n-1}$ is the second-order difference, and $\mathrm{V}^{n} x_{t-n-1}$ is the $n^{\text {th }}$-order difference.

In a $2 \mathrm{D}$ plane, the location data of nodes in the $(x, y)$ direction is processed to estimate the location of the moving target. Through formulas (5) and (6), the moving method and speed of the node at time $t$ are respectively obtained as follows:

$\hat{\alpha}_{t}=\left\{\begin{array}{l}\arctan \left(\frac{\hat{y}_{t}-y_{t-1}}{\hat{x}_{t}-x_{t-1}}, \quad \hat{x}_{t} \geq x_{t-1}\right. \\ \arctan \left(\frac{\hat{y}_{t}-y_{t-1}}{\hat{x}_{t}-x_{t-1}}\right)+\pi, \hat{x}_{t}<x_{t-1}\end{array}\right.$

$\hat{v}_{t}=\sqrt{\left(\hat{x}_{t}-x_{t-1}\right)^{2}+\left(\hat{y}_{t}-y_{t-1}\right)^{2}}$

The location prediction function of the node at time $t$ is shown in formula (7):

$\left[\begin{array}{c}x_{t}^{i} \\ y_{t}^{i}\end{array}\right]=\left[\begin{array}{c}x_{t-1}^{i}+v_{t} \cos \left(\alpha_{t}\right) \Delta t \\ y_{t-1}^{i}+v_{t} \sin \left(\alpha_{t}\right) \Delta t\end{array}\right]$

where $v_{t} \in\left[0.6 v_{t}, 1.4 \hat{v}_{t}\right], \quad \alpha_{t} \in\left[\hat{\alpha}_{t}-\pi / 6, \hat{\alpha}_{t}+\pi / 6\right]$.

If the number of nodes in the dynamic coverage group formed in the target is $n$, then the maximum possible area that the target may reach is determined by formula (8):

$$
\left\{\left(\max _{i=1}^{n}\left(x_{j}-r_{c}\right), \min _{i=1}^{n}\left(x_{j}+r_{c}\right)\right),\left(\max _{i=1}^{n}\left(y_{j}-r_{c}\right), \min _{i=1}^{n}\left(y_{j}+r_{c}\right)\right)\right\}
$$

Where $\left(x_{i}, y_{i}\right)$ is the location coordinates of nodes.

\subsection{Node data reporting frequency}

The data reception and transmission frequency of cluster head nodes in the dynamic coverage group should dynamically change because of the moving speed change of the moving target. Internet energy utilization efficiency and the accuracy of data acquisition in the dynamic coverage process is improved by participating in the target dynamic coverage on the basis of the speed size of the target. The final message of the target and member node stored by the original cluster head node is sent to the current cluster head node. By the target location algorithm of two cluster head nodes, the average moving speed of the moving target is calculated in this period, as shown in formula (9):

$$
v_{a v g}=\left|L_{c u r}-L_{p r e}\right| /\left(t_{c u r}-t_{p r e}\right)
$$

For the average moving speed of target, the frequency of data dynamically transmitted by the cluster head node is set using formula (10):

$$
f=\left\lfloor\frac{\bar{v}}{d_{t h}}\right\rfloor+1
$$

Where $d_{t h}$ denotes the maximum moving distance of a moving target reported by the cluster head nodes for two times.

\subsection{Algorithm description}

The algorithm of self-adapting moving target coverage is described as follows:

Step 1: The network is initiated and divided on the basis of the communication radius of the sensor nodes.

Step 2: The cluster head node monitors the moving target and awakens its member nodes in the waiting state when the moving target enters the monitoring area.

Step 3: The weights of participation nodes are calculated to allow the nodes that meet the correct participation requirements to enter the active state and construct the initial dynamic coverage group.

Step 4: The cluster head nodes collect the target data transmitted to the base station after the fusion process.

Step 5: If a new node is added to the dynamic coverage group or the node exits the coverage group, then the dynamic coverage group is reconstructed, and the new cluster head node is selected. Otherwise, step 4 is adopted for execution.

Step 6: If the coverage group is exited, the original cluster head node sends the target node location and members to the new cluster head node and then shifts to the sleeping state.

Step 7: If the target node is in the monitoring area, then step 4 is adopted; conversely, the monitoring task is completed.

\section{Result Analysis and Discussion}

4.1 Parameter setting

The simulation experiment is designed on the 
communication simulation platform OMNET ++4.5 on the basis of the performance evaluation index to evaluate the performance of the dynamic coverage algorithm. In the experimental environment, the sensor nodes are randomly deployed in a target monitoring area of $500 \times 500 \mathrm{~m}^{2}$ and divided into a grid with an area of $17 \times 17 \mathrm{~m}^{2}$. The cluster head node in each grid is ensured to directly communicate with eight adjacent cluster head nodes [4]. The moving speed of the target node can be $5,10,15,10$, and $25 \mathrm{~m} / \mathrm{s}$. The main parameters are set as shown in Table 1 .

Table 1. Simulation parameters

\begin{tabular}{l|c}
\hline Parameter & Value \\
\hline Field size: $l(m)$ & $500 \times 500$ \\
Number of nodes: $N_{s}$ & 800 \\
Sensing radius of node: $r_{s}(m)$ & 50 \\
Communication radius of sensor: $r_{c}(m)$ & 50 \\
Speed of a mobile target: $v(m / s)$ & $5,10,15,20,25$ \\
Data packet: $(b)$ & 1000 \\
Participation threshold: $\left(w_{t h r}\right)$ & $2,3,4,5$ \\
\hline
\end{tabular}

\subsection{Evaluation index}

Definition 4 (average location error): In a time period of $T$, the location accuracy of moving target by network nodes, that is the average location error $Q$, is given as :

$$
\begin{aligned}
& q(t)=\sqrt{\left(x_{e s t}(t)-x_{a c t}(t)\right)^{2}+\left(y_{e s t}(t)-y_{a c t}(t)\right)^{2}} \\
& Q=\frac{1}{T} \int_{0}^{T} q(t) d t
\end{aligned}
$$

Where $q(t)$ denotes the location error of target at time $t$, $\left(x_{\text {est }}(t), y_{\text {est }}(t)\right)$ is the estimated location of the target at time $t$ and $\left(x_{a c t}(t), y_{a c t}(t)\right)$ represents the real location of the target at time $t$.

Definition 5 (average energy consumption): The energy utilization ratio of a network node is obtained as follows:

$$
E_{\text {avg }}=\frac{1}{N_{s}} \sum_{1}^{N_{s}} e_{i}
$$

Where $e_{i}$ denotes the energy consumption of participation node $s_{i}$ and $N_{s}$ represents the number of nodes that participate in moving target monitoring.

\subsection{Experimental result analysis}

On the basis of the experimental evaluation index, a comparative analysis of the average location error, average energy consumption, and network overall energy consumption was conducted depending on different participation thresholds.

(1) Average location error at different target speeds Under the condition of same participation threshold $w_{t h r}$ (Figure 2), the location error shows an increase trend because the average time of moving target monitoring by the node decreases owing to the increased reconstruction speed of the dynamic coverage group with the increased target moving speed. At the same moving speed, location error evidently increases due to the decrease in participation nodes with the increase in participation threshold. The threshold of the node that participates in monitoring decreases, the nodes participating in the target monitoring increase, and the location error of the nodes for the moving target is significantly reduced with notable accuracy improvement. Based on quantitative calculation, the average location error of participation node for the target decreases by about $56.7 \%$ when the participation monitoring threshold decreases from 5 to 2 . Therefore, appropriate node participation monitoring threshold can be selected on the basis of the location accuracy requirements of a moving target in practical application.

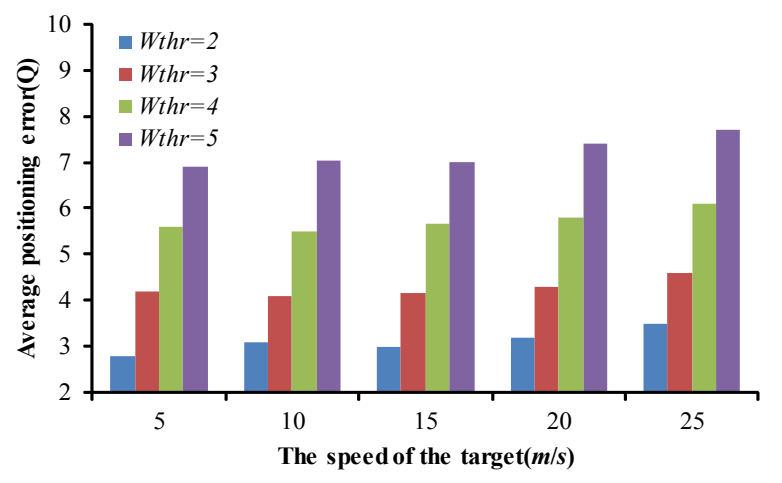

Fig. 2. Average location error

(2) Average energy consumption comparison at different speeds

By comparing the average energy consumption of the moving target at different speeds (Figure 3), the number of nodes that participate in the target monitoring decreases in accordance with an increasingly large threshold $w_{t h r}$ of the node involved in monitoring, and additional nodes are in the sleeping state. Under these conditions, network energy is conserved, and the network average energy consumption rapidly decreases. Under identical participation threshold conditions, node data reporting frequency and node average energy consumption increase according to increasing moving target speed. With a relatively low participation threshold, such as $w_{t h r}=2$, this result shows that the nodes near the target basically participate in the monitoring of the target node, and the difference in network average energy consumption is smaller than that under the condition without a preset threshold. When the node participation threshold is set at $w_{t h r}=3$, the average energy consumption decreases by $55 \%$ relative to consumption without a preset threshold. Therefore, the network average energy consumption can be effectively reduced to improve the network life cycle by setting an appropriate node participation threshold.

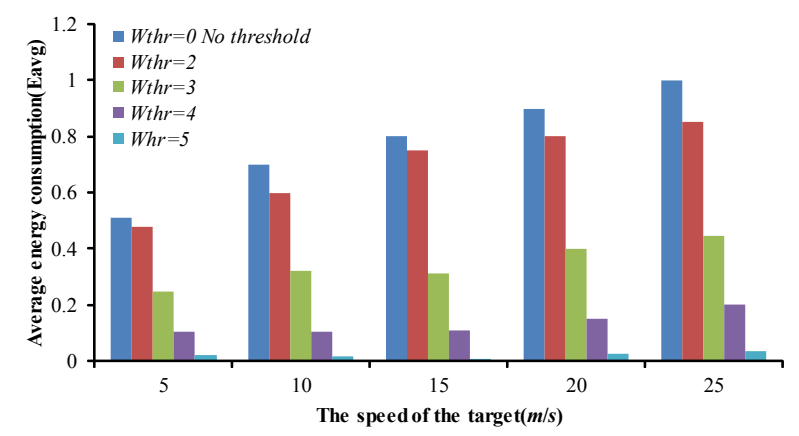

Fig. 3. Average energy consumption 
(3) Influence of target location prediction model on the network energy consumption

The influence of the target location prediction model on network energy consumption is depicted in Figure 4. When the participation threshold is set to 3 and 5, the use of the target location prediction model significantly reduces the network overall energy consumption. For instance, at $w_{t h r}=2$, the location prediction model can decrease the network average overall energy consumption by $41 \%$ and even up to $46 \%$. This result shows that the node prediction model can effectively predict the target moving location, thereby diminishing the number of redundant nodes that participate in monitoring calculations and reducing network energy consumption.

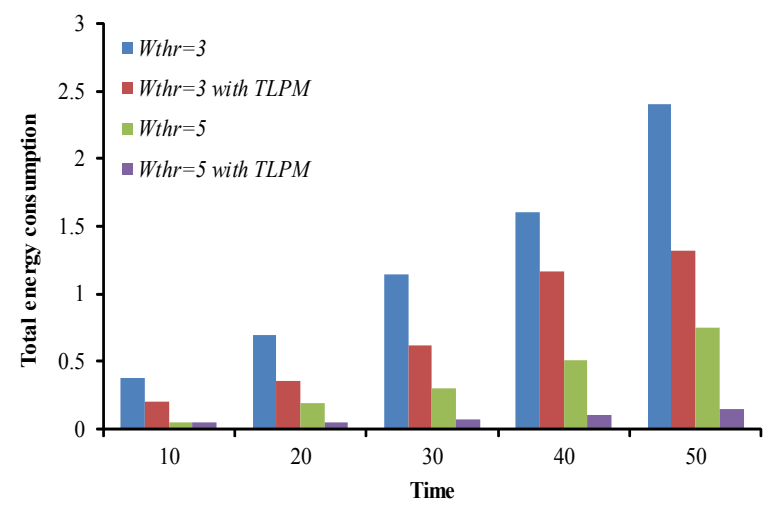

Fig. 4. Influence of TLPM on network energy consumption

The above experimental analysis implies that setting an appropriate threshold yields an augmented difference in controlling the node location accuracy and network energy consumption. The threshold-controllable algorithm for moving target coverage can play an effective role in moving target location accuracy and network average energy consumption by constructing a dynamic coverage group, establishing a node state scheduling strategy, formulating the moving target location prediction model, and elucidating the node data reporting frequency self-adjusting mechanism. As such, the algorithm is suitable for application in wireless sensor network environments with limited node energy.

\section{Conclusion}

To improve the location accuracy and network energy utilization of moving targets in a wireless sensor network, we established a moving target coverage model. We also analyzed the influencing factors of the node participation threshold on the moving target location prediction accuracy and network node energy consumption through thresholdcontrollable node participation. The following conclusions could be obtained:

(1) Selection of appropriate nodes that participate in the dynamic coverage of a moving target can improve moving target location accuracy and reflect the influence of node participation threshold on the moving target location accuracy.

(2) Increased node participation threshold can reduce the number of nodes involved in monitoring and diminish network energy consumption. However, this approach can affect target location accuracy. Therefore, the appropriate participation threshold must be selected for target location accuracy and network energy consumption.

(3) Triggering of participation nodes in advance when the movement locus of moving targets is predicted to not only improve the real-time monitoring capability and reduce the number of unnecessary participation nodes but also decrease network energy consumption and enhance the network life cycle.

After considering the total process of real-time monitoring of the wireless sensor network, we found that different target moving speeds and various node participation thresholds markedly influence moving target location accuracy and network energy consumption. In conclusion, the threshold-controllable algorithm for moving target coverage can provide a feasible solution and theoretical guidance for moving target coverage in wireless sensor networks. However, numerous problems remain to be explored in this field. Examples of future research topics include the approach for establishing the perception model of coverage, optimization of the coverage algorithm, reasonable evaluation of network coverage quality, and reduction of node location error in the environment with $3 \mathrm{D}$ features.

\section{Acknowledgements}

This work was supported by the Key Scientific Research Project of Universities and Colleges in Henan (Grant Nos. 18B520026 and 17A520044), the Young Backbone Teacher Program of Universities and Colleges in Henan (Grant No. 2016GGJS-158), the Luoyang Institute of Science and Technology High-level Research Start Foundation (Grant No. 2017BZ07) and the Natural Science and Technology Research of Henan Province Department of Science Foundation (Grant No. 182102210428).

This is an Open Access article distributed under the terms of the Creative Commons Attribution License

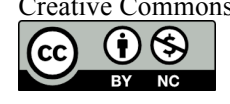

\section{References}

1. Jin, Y., Ding, Y., Hao, K., Jin, Y., “An endocrine-based intelligent distributed cooperative algorithm for target tracking in wireless sensor networks". Soft Computing, 19(5), 2015, pp. 1427-1441.

2. Wang, B., "Coverage problems in sensor networks: A survey". Acm Computing Surveys, 43(4), 2011, pp.1-53.

3. Sangwan, A., Singh, R. P., "Survey on Coverage Problems in Wireless Sensor Networks". Wireless Personal Communications, 80(4), 2015, pp.1475-1500

4. Sun, Z., Zhao, G., Xing, X., "ENCP: a new energy-efficient nonlinear coverage control protocol in mobile sensor networks". Eurasip Journal on Wireless Communications \& Networking, 2018(1), 2018, pp.1-15.
5. Yong, H. K., Min, S.P., Jin, B. P., Yoon, T. S., "TDOA Based Moving Target Velocity Estimation in Sensor Network". Transactions of the Korean Institute of Electrical Engineers, 64(3), 2015, pp.445-450.

6. Sun, Z., Zhang, Y., Nie, Y., Wei, W., Lloret, J., Song, H., "CASMOC: a novel complex alliance strategy with multi-objective optimization of coverage in wireless sensor networks". Wireless Networks, 23(4), 2017, pp.1201-1222.

7. Xu, B., Zhu, Y., Kim, D., Jiang, H., Tokuta, A. Q., "Strengthening barrier-coverage of static sensor network with mobile sensor nodes". Wireless Networks, 22(1), 2016, pp.1-10. 
8. Mostafaei, H., Shojafar, M., Zaher, B., Zaher, B., Singhal, M. "Barrier coverage of WSNs with the imperialist competitive algorithm”. Journal of Supercomputing, (12), 2017, pp.1-24.

9. Tao, D., Mao, X. F., Wu, H., "Barrier Coverage Algorithm for Moving Target in Directional Sensor Networks". Journal of Beijing University of Posts \& Telecommunications, 36(5), 2013, pp.6-9.

10. Zhang, M. J., Cui, J., University, L. T., "Prediction Model of Moving Target Tracking in Wireless Sensor Networks". Computer Systems \& Applications, 3(3), 2014, pp.258-271.

11. Raja, S., "A Quantitative Analysis of Algorithms for Energy Efficient Coverage in WSN". International Journal of Computer Applications, 95(5), 2014, pp.29-33.

12. Zebbane, B., Chenait, M., Badache, N., "A Group-Based EnergySaving Algorithm for Sleep/Wake Scheduling and Topology Control in Wireless Sensor Networks". Wireless Personal Communications, 84(2), 2015, pp.959-983.

13. Li, Y., Zheng, Y., Liu, Y. H., "Energy Saving Target Tracking Using Mobile Sensor Networks". Journal of automation, 33(11), 2007, pp.1156-1162.

14. Liu, J., Liu, H., Ye, N., Cui, Z., "Adaptive Dynamic-Cluster Based Target Tracking Strategy in Wireless Sensor Networks". Journal of Northeastern University(Natural Science), 32(8), 2011, pp.10801083.

15. Ren, Q. Q., Li, J. Z., Gao, H., Cheng, S. Y., “A Two-Phase Sleep Scheduling Based Protocol for Target Tracking in Sensor Networks". Chinese Journal of Computers, 32(10), 2009, pp.19711979.
16. Yuan, G., Sun, P., Zhao, J., Li, D., Wang, C., "A review of moving object trajectory clustering algorithms". Artificial Intelligence Review, 47(1), 2017, pp.123-144.

17. Ez-Zaidi, A., Rakrak, S., "A Comparative Study of Target Tracking Approaches in Wireless Sensor Networks". Journal of Sensors, 2016(2), 2015, pp.1-11.

18. Sun, X. Y., Li, J. D., Huang, P. Y., Zhang, W. Z., "Distance-based target tracking algorithm in binary sensor network". Journal on Communications, 31(12), 2010, pp.140-146.

19. Zhang, W., Cao, G., "DCTC: dynamic convoy tree-based collaboration for target tracking in sensor networks". IEEE Transactions on Wireless Communications, 3(5), 2004, pp.1689. 1701.

20. Sahoo, P. K., Sheu, J. P., Hsieh, K. Y., "Target tracking and boundary node selection algorithms of wireless sensor networks for internet services". Information Sciences, 230(1), 2013, pp.21-38.

21. Xing, X., Wang, G., Li, J., "Collaborative Target Tracking in Wireless Sensor Networks". Ad Hoc \& Sensor Wireless Networks, 23(1), 2014, pp.117-135.

22. Velmani, R., Kaarthick, B., "An Efficient Cluster-Tree Based Data Collection Scheme for Large Mobile Wireless Sensor Networks". IEEE Sensors Journal, 15(4), 2015, pp.2377-2390.

23. Wang, G., Bhuiyan, M. Z. A., Cao, J, Wu, J., "Detecting Movements of a Target Using Face Tracking in Wireless Sensor Networks". IEEE Transactions on Parallel \& Distributed Systems, 25(4), 2014, pp.939-949.

24. Bhowmik, S., Giri, C., "Convoy Tree Based Fuzzy Target Tracking in Wireless Sensor Network". International Journal of Wireless Information Networks, 24(9), 2017, pp.1-9. 